TAMKANG JOURNAL OF MATHEMATICS

Volume 33, Number 4, Winter 2002

\title{
A GENERAL OSTROWSKI TYPE INEQUALITY FOR DOUBLE INTEGRALS
}

\author{
G. HANNA, S. S. DRAGOMIR AND P. CERONE
}

\begin{abstract}
Some generalisations of an Ostrowski Type Inequality in two dimensions for $n$-time differentiable mappings are given. The result is an Integral Inequality with bounded $n$-time derivatives. This is employed to approximate double integrals using one dimensional integrals and function evaluations at the boundary and interior points.
\end{abstract}

\section{Introduction}

The classical Ostrowski Integral Inequality (see [2], p.468) in one dimension stipulates a bound between a function evaluated at an interior point $x$ and the average of the function $f$ over an interval. That is,

$$
\left|f(x)-\frac{1}{b-a} \int_{a}^{b} f(t) d t\right| \leq\left[\frac{1}{4}+\frac{\left(x-\frac{a+b}{2}\right)^{2}}{(b-a)^{2}}\right](b-a)\left\|f^{\prime}\right\|_{\infty}
$$

for all $x \in[a, b]$, where $f^{\prime} \in L_{\infty}(a, b)$ and $f:[a, b] \rightarrow \mathbb{R}$ is a differentiable mapping on $(a, b)$.

Here, the constant $\frac{1}{4}$ is sharp in the sense that it cannot be replaced by a smaller constant. We also observe that the tightest bound is obtained at $x=\frac{a+b}{2}$, resulting in the well-known mid-point inequality. In [1], P. Cerone, S. S. Dragomir and J. Roumeliotis proved the following Ostrowski type inequality for $n$-time differentiable mappings.

Theorem 1. Let $f:[a, b] \rightarrow \mathbb{R}$ be a mapping such that $f^{(n-1)}$ is absolutely continuous on $[a, b]$ and $f^{(n)} \in L_{\infty}[a, b]$. Then for all $x \in[a, b]$, we have the inequality:

$$
\begin{aligned}
& \left|\int_{a}^{b} f(t) d t-\sum_{k=0}^{n-1}\left[\frac{(b-x)^{k+1}+(-1)^{k}(x-a)^{k+1}}{(k+1) !}\right] f^{(k)}(x)\right| \\
\leq & \frac{\left\|f^{(n)}\right\|_{\infty}}{(n+1) !}\left[(x-a)^{n+1}+(b-x)^{n+1}\right] \leq \frac{\left\|f^{(n)}\right\|_{\infty}(b-a)^{n+1}}{(n+1) !}
\end{aligned}
$$

where $\left\|f^{(n)}\right\|_{\infty}:=\sup _{t \in[a, b]}\left|f^{(n)}(t)\right|<\infty$.

Received September 14, 2001; revised November 15, 2001.

2000 Mathematics Subject Classification. 26D15, 41A55.

Key words and phrases. Ostrowski inequality, double integrals, Lebesgue norms. 
For other similar results for $n$-time differentiable mappings, see the paper [7] by Fink and [8] by Anastassiou.

In [3] and [4] the authors proved some inequalities of Ostrowski type for double integrals in terms of different norms.

In this paper we combine the above two results and develop them in two dimensions to obtain a generalization of the Ostrowski inequality for $n$-time differentiable mappings using different types of norms.

\section{Integral Identities}

The result presented here approximates a two-dimensional integral for $n-$ time differentiable mappings via the application of one dimensional integrals at the boundary, function evaluations at interior or boundary points and $\backslash$ or its derivatives at a multiple number of points over the given region.

The following result holds.

Theorem 2. Let $f:[a, b] \times[c, d] \rightarrow \mathbb{R}$ be a continuous mapping such that the following partial derivatives $\frac{\partial^{l+k} f(\cdot, \cdot)}{\partial t^{k} \partial s^{l}}, k=0,1, \ldots, n-1, l=0,1, \ldots, m-1$ exist and are continuous on $[a, b] \times[c, d]$. Further, for $K_{n}:[a, b]^{2} \rightarrow \mathbb{R}, S_{m}:[c, d]^{2} \rightarrow \mathbb{R}$ given by

$$
K_{n}(x, t):=\left\{\begin{array}{ll}
\frac{(t-a)^{n}}{n !}, & t \in[a, x] \\
\frac{(t-b)^{n}}{n !}, & t \in(x, b]
\end{array}, \quad S_{m}(y, s):= \begin{cases}\frac{(s-c)^{m}}{m !}, & s \in[c, y] \\
\frac{(s-d)^{m}}{m !}, & s \in(y, d]\end{cases}\right.
$$

then for all $(x, y) \in[a, b] \times[c, d]$, we have the identity:

$$
\begin{aligned}
\int_{a}^{b} \int_{c}^{d} f(t, s) d s d t= & \sum_{k=0}^{n-1} \sum_{l=0}^{m-1} X_{k}(x) Y_{l}(y) \frac{\partial^{l+k} f(x, y)}{\partial t^{k} \partial s^{l}} \\
& +(-1)^{m} \sum_{k=0}^{n-1} X_{k}(x) \int_{c}^{d} S_{m}(y, s) \frac{\partial^{k+m} f(x, s)}{\partial t^{k} \partial s^{m}} d s \\
& +(-1)^{n} \sum_{l=0}^{m-1} Y_{l}(y) \int_{a}^{b} K_{n}(x, t) \frac{\partial^{n+l} f(t, y)}{\partial t^{n} \partial s^{l}} d t \\
& +(-1)^{m+n} \int_{a}^{b} \int_{c}^{d} K_{n}(x, t) S_{m}(y, s) \frac{\partial^{n+m} f(t, s)}{\partial t^{n} \partial s^{m}} d s d t
\end{aligned}
$$

where

$$
X_{k}(x)=\frac{(b-x)^{k+1}+(-1)^{k}(x-a)^{k+1}}{(k+1) !}, \quad Y_{l}(y)=\frac{(d-y)^{l+1}+(-1)^{l}(y-c)^{l+1}}{(l+1) !} .
$$


Proof. Applying the identity (see [1])

$$
\int_{a}^{b} g(t) d t=\sum_{k=0}^{n-1}\left[\frac{(b-x)^{k+1}+(-1)^{k}(x-a)^{k+1}}{(k+1) !}\right] g^{(k)}(x)+(-1)^{n} \int_{a}^{b} P_{n}(x, t) g^{(n)}(t) d t
$$

where

$$
P_{n}(x, t)= \begin{cases}\frac{(t-a)^{n}}{n !} & \text { if } t \in[a, x], \\ \frac{(t-b)^{n}}{n !} & \text { if } t \in(x, b]\end{cases}
$$

which has been used essentially in the proof of Theorem 1 , for the partial mapping $f(\cdot, s)$, $s \in[c, d]$, we can write

$$
\begin{aligned}
\int_{a}^{b} f(t, s) d t= & \sum_{k=0}^{n-1}\left[\frac{(b-x)^{k+1}+(-1)^{k}(x-a)^{k+1}}{(k+1) !}\right] \frac{\partial^{k} f(x, s)}{\partial t^{k}} \\
& +(-1)^{n} \int_{a}^{b} K_{n}(x, t) \frac{\partial^{n} f(t, s)}{\partial t^{n}} d t
\end{aligned}
$$

for every $x \in[a, b]$ and $s \in[c, d]$.

Integrating (2.5) over $s$ on $[c, d]$, we deduce

$$
\begin{aligned}
\int_{a}^{b} \int_{c}^{d} f(t, s) d s d t= & \sum_{k=0}^{n-1}\left[\frac{(b-x)^{k+1}+(-1)^{k}(x-a)^{k+1}}{(k+1) !}\right] \int_{c}^{d} \frac{\partial^{k} f(x, s)}{\partial t^{k}} d s \\
& +(-1)^{n} \int_{a}^{b} K_{n}(x, t)\left(\int_{c}^{d} \frac{\partial^{n} f(t, s)}{\partial t^{n}} d s\right) d t
\end{aligned}
$$

for all $x \in[a, b]$.

Applying the identity (2.4) again for the partial mapping $\frac{\partial^{k} f(x, \cdot)}{\partial t^{k}}$ on $[c, d]$, we obtain

$$
\begin{aligned}
\int_{c}^{d} \frac{\partial^{k} f(x, s)}{\partial t^{k}} d s= & \sum_{l=0}^{m-1}\left[\frac{(d-y)^{l+1}+(-1)^{l}(y-c)^{l+1}}{(l+1) !}\right] \frac{\partial^{l}}{\partial s^{l}}\left(\frac{\partial^{k} f(x, y)}{\partial t^{k}}\right) \\
& +(-1)^{m} \int_{c}^{d} S_{m}(y, s) \frac{\partial^{m}}{\partial s^{m}}\left(\frac{\partial^{k} f(x, s)}{\partial t^{k}}\right) d s \\
= & \sum_{l=0}^{m-1}\left[\frac{(d-y)^{l+1}+(-1)^{l}(y-c)^{l+1}}{(l+1) !}\right] \frac{\partial^{l+k} f(x, y)}{\partial t^{k} \partial s^{l}} \\
& +(-1)^{m} \int_{c}^{d} S_{m}(y, s) \frac{\partial^{k+m} f(x, s)}{\partial t^{k} \partial s^{m}} d s .
\end{aligned}
$$


In addition, the identity (2.4) applied for the partial derivative $\frac{\partial^{n} f(t, \cdot)}{\partial t^{n}}$ also gives

$$
\begin{aligned}
\int_{c}^{d} \frac{\partial^{n}(t, s)}{\partial t^{n}} d s= & \sum_{l=0}^{m-1}\left[\frac{(d-y)^{l+1}+(-1)^{l}(y-c)^{l+1}}{(l+1) !}\right] \frac{\partial^{n+l} f(t, y)}{\partial t^{n} \partial y^{l}} \\
& +(-1)^{m} \int_{c}^{d} S_{m}(y, s) \frac{\partial^{n+m} f(t, s)}{\partial t^{n} \partial s^{m}} d s .
\end{aligned}
$$

Using (2.7) and (2.8) and substituting into (2.6) will produce the result (2.2), and thus the theorem is proved.

Utilising the result (2.2) we will produce the midpoint cubature rule for two - dimensional rectangular region for $n$-times differentiable mappings as well as the trapezoid cubature rule as shown in the following two corollaries respectively.

Corollary 1. With the assumptions as in Theorem 2, we have the representation

$$
\begin{aligned}
& \int_{a}^{b} \int_{c}^{d} f(t, s) d s d t \\
= & \sum_{k=0}^{n-1} \sum_{l=0}^{m-1} X_{k}\left(\frac{a+b}{2}\right) Y_{l}\left(\frac{c+d}{2}\right) \frac{\partial^{l+k} f\left(\frac{a+b}{2}, \frac{c+d}{2}\right)}{\partial t^{k} \partial s^{l}} \\
& +(-1)^{m} \sum_{k=0}^{n-1} X_{k}\left(\frac{a+b}{2}\right) \int_{c}^{d} \tilde{S}_{m}(s) \frac{\partial^{k+m} f\left(\frac{a+b}{2}, s\right)}{\partial t^{k} \partial s^{m}} d s \\
& +(-1)^{n} \sum_{l=0}^{m-1} Y_{l}\left(\frac{c+d}{2}\right) \int_{a}^{b} \tilde{K}_{n}(t) \frac{\partial^{n+l} f\left(t, \frac{c+d}{2}\right)}{\partial t^{n} \partial s^{l}} d t \\
& +(-1)^{m+n} \int_{a}^{b} \int_{c}^{d} \tilde{K}_{n}(t) \tilde{S}_{m}(s) \frac{\partial^{n+m} f(t, s)}{\partial t^{n} \partial s^{m}} d s d t,
\end{aligned}
$$

where $X_{k}(\cdot)$ and $Y_{l}(\cdot)$ are as given in $(2.3)$ and so

$$
X_{k}\left(\frac{a+b}{2}\right)=\left[\frac{1+(-1)^{k}}{(k+1) !}\right] \frac{(b-a)^{k+1}}{2^{k+1}}, \quad Y_{l}\left(\frac{c+d}{2}\right)=\left[\frac{1+(-1)^{l}}{(l+1) !}\right] \frac{(d-c)^{l+1}}{2^{l+1}}
$$

and $\tilde{K}_{n}:[a, b] \rightarrow \mathbb{R}, \tilde{S}_{m}:[c, d] \rightarrow \mathbb{R}$ are given by

and

$$
\tilde{K}_{n}(t)=K_{n}\left(\frac{a+b}{2}, t\right)
$$

$$
\tilde{S}_{m}(s)=S_{m}\left(\frac{c+d}{2}, s\right)
$$

on using (2.1). 
Corollary 2. Let $f$ be as in Theorem 2. Then we have the following identity

$$
\begin{aligned}
& \int_{a}^{b} \int_{c}^{d} f(t, s) d s d t \\
= & \frac{1}{4} \sum_{k=0}^{n-1} \sum_{l=0}^{m-1}\left[\frac{(b-a)^{k+1}}{(k+1) !}\right] \times\left[\frac{(d-c)^{l+1}}{(l+1) !}\right] \\
& \times \frac{\partial^{l+k}}{\partial t^{k} \partial s^{l}}\left[f(a, c)+(-1)^{l} f(a, d)+(-1)^{k} f(b, c)+(-1)^{l+k} f(b, d)\right] \\
& +\frac{1}{4}(-1)^{m} \sum_{k=0}^{n-1}\left[\frac{(b-a)^{k+1}}{(k+1) !}\right] \times\left[\int_{c}^{d} \frac{(s-c)^{m}+(s-d)^{m}}{m !} \frac{\partial^{k+m}}{\partial t^{k} \partial s^{m}}\left[f(a, s)+(-1)^{k} f(b, s)\right] d s\right] \\
& +\frac{1}{4}(-1)^{n} \sum_{l=0}^{m-1}\left[\frac{(d-c)^{l+1}}{(l+1) !}\right] \times\left[\int_{a}^{b} \frac{(t-a)^{n}+(t-b)^{n}}{n !} \frac{\partial^{n+l}}{\partial t^{n} \partial s^{l}}\left[f(t, c)+(-1)^{k} f(t, d)\right] d t\right] \\
& +\frac{1}{4}(-1)^{m+n} \int_{a}^{b} \int_{c}^{d} \frac{(s-c)^{m}+(s-d)^{m}}{m !} \cdot \frac{(t-a)^{n}+(t-b)^{n}}{n !} \frac{\partial^{n+m} f(t, s)}{\partial t^{n} \partial s^{m}} d s d t, \quad(2.10)
\end{aligned}
$$

where $X_{n-1}(t)$ and $Y_{m-1}(s)$ are as given by (2.3).

Proof. By substituting $(x, y)=(a, c),(a, d),(b, c),(b, d)$ respectively and summing the resulting identities and after some simplification, we get the desired inequality (2.10).

In the above section we promoted some two-dimensional integral identities for $n$-times differentiable mappings, which are useful in themselves. They are exploited in the next section to obtain two-dimensional integral inequalities on Lebesgue spaces $L_{\infty}\left[\left[a_{1}, b_{1}\right] \times\right.$ $\left.\left[a_{2}, b_{2}\right]\right], L_{p}\left[\left[a_{1}, b_{1}\right] \times\left[a_{2}, b_{2}\right]\right]$ and $L_{1}\left[\left[a_{1}, b_{1}\right] \times\left[a_{2}, b_{2}\right]\right]$.

\section{Some Integral Inequalities}

In this section we tap the equalities of Section 2 and develop inequalities for the depiction of the two-dimensional integral of a function with respect to one-dimensional integrals at the boundary, function evaluations at interior or boundary points and/or derivatives at a multiple number of points over the entire region. We start with the following result

Theorem 3. Let $f:[a, b] \times[c, d] \rightarrow \mathbb{R}$ be continuous on $[a, b] \times[c, d]$, and assume that $\frac{\partial^{n+m} f}{\partial t^{n} \partial s^{m}}$ exist on $(a, b) \times(c, d)$. Then we have the inequality

$$
\begin{aligned}
& \mid \int_{a}^{b} \int_{c}^{d} f(t, s) d s d t-\sum_{k=0}^{n-1} \sum_{l=0}^{m-1} X_{k}(x) \cdot Y_{l}(y) \frac{\partial^{l+k} f(x, y)}{\partial t^{k} \partial s^{l}} \\
& -(-1)^{m} \sum_{k=0}^{n-1} X_{k}(x) \int_{c}^{d} S_{m}(y, s) \frac{\partial^{k+m} f(x, s)}{\partial t^{k} \partial s^{m}} d s-(-1)^{n} \sum_{l=0}^{m-1} Y_{l}(y) \int_{a}^{b} K_{n}(x, t) \frac{\partial^{n+l} f(t, y)}{\partial t^{n} \partial s^{l}} d t \mid
\end{aligned}
$$




$$
\leq\left\{\begin{array}{l}
\frac{1}{(n+1) !(m+1) !}\left[(x-a)^{n+1}+(b-x)^{n+1}\right] \times\left[(y-c)^{m+1}+(d-y)^{m+1}\right] \times\left\|\frac{\partial^{n+m} f}{\partial t^{n} \partial s^{m}}\right\|_{\infty} \\
\text { if } \frac{\partial^{n+m} f}{\partial t^{n} \partial s^{m}} \in L_{\infty}([a, b] \times[c, d]) ; \\
\frac{1}{n ! m !}\left[\frac{(x-a)^{n q+1}+(b-x)^{n q+1}}{n q+1}\right]^{\frac{1}{q}} \times\left[\frac{(y-c)^{m q+1}+(d-y)^{m q+1}}{m q+1}\right]^{\frac{1}{q}} \times\left\|\frac{\partial^{n+m} f}{\partial t^{n} \partial s^{m}}\right\|_{p} \\
\text { if } \frac{\partial^{n+m} f}{\partial t^{n} \partial s^{m}} \in L_{p}([a, b] \times[c, d]), \quad p>1, \quad \frac{1}{p}+\frac{1}{q}=1 \\
\frac{1}{4 n ! m !}\left[(x-a)^{n}+(b-x)^{n}+\left|(x-a)^{n}-(b-x)^{n}\right|\right] \\
\quad \times\left[(y-c)^{m}+(d-y)^{m}+\left|(y-c)^{m}-(d-y)^{m}\right|\right] \times\left\|\frac{\partial^{n+m} f}{\partial t^{n} \partial s^{m}}\right\|_{l} \\
\text { if } \frac{\partial^{n+m} f}{\partial t^{n} \partial s^{m}} \in L_{1}([a, b] \times[c, d])
\end{array}\right.
$$

for all $(x, y) \in[a, b] \times[c, d]$, where

$$
\begin{aligned}
\left\|\frac{\partial^{n+m} f}{\partial t^{n} \partial s^{m}}\right\|_{\infty} & =\sup _{(t, s) \in[a, b] \times[c, d]}\left|\frac{\partial^{n+m} f(t, s)}{\partial t^{n} \partial s^{m}}\right|<\infty \\
\left\|\frac{\partial^{n+m} f}{\partial t^{n} \partial s^{m}}\right\|_{p} & =\left(\int_{a}^{b} \int_{c}^{d}\left|\frac{\partial^{n+m}}{\partial t^{n} \partial s^{m}} f(t, s)\right|^{p} d t d s\right)^{\frac{1}{p}}<\infty .
\end{aligned}
$$

Proof. Using Theorem 2, we get from (2.2)

$$
\begin{aligned}
& \mid \int_{a}^{b} \int_{c}^{d} f(t, s) d s d t-\sum_{k=0}^{n-1} \sum_{l=0}^{m-1} X_{k}(x) \cdot Y_{l}(y) \frac{\partial^{l+k} f(x, y)}{\partial t^{k} \partial s^{l}} \\
& -(-1)^{m} \sum_{k=0}^{n-1} X_{k}(x) \int_{c}^{d} S_{m}(y, s) \frac{\partial^{k+m} f(x, s)}{\partial t^{k} \partial s^{m}} d s \\
& \quad-(-1)^{n} \sum_{l=0}^{m-1} Y_{l}(y) \int_{a}^{b} K_{n}(x, t) \frac{\partial^{n+l} f(t, y)}{\partial t^{n} \partial s^{l}} d t \mid \\
& =\left|\int_{a}^{b} \int_{c}^{d} K_{n}(x, t) S_{m}(y, s) \frac{\partial^{n+m} f(t, s)}{\partial t^{n} \partial s^{n}} d s d t\right| \\
& \leq \int_{a}^{b} \int_{c}^{d}\left|K_{n}(x, t) S_{m}(y, s)\right|\left|\frac{\partial^{n+m} f(t, s)}{\partial t^{n} \partial s^{n}}\right| d s d t .
\end{aligned}
$$

Using Hölder's inequality and properties of the modulus and integral, then we have that 


$$
\begin{aligned}
& \int_{a}^{b} \int_{c}^{d}\left|K_{n}(x, t) S_{m}(y, s)\right|\left|\frac{\partial^{n+m} f(t, s)}{\partial t^{n} \partial s^{m}}\right| d s d t \\
\leq & \left\{\begin{array}{l}
\left\|\frac{\partial^{n+m} f}{\partial t^{n} \partial s^{m}}\right\|_{\infty} \int_{a}^{b} \int_{c}^{d}\left|K_{n}(x, t) S_{m}(y, s)\right| d t d s \\
\left\|\frac{\partial^{n+m} f}{\partial t^{n} \partial s^{m}}\right\|_{p}\left(\int_{a}^{b} \int_{c}^{d}\left|K_{n}(x, t) S_{m}(y, s)\right|^{q} d t d s\right)^{\frac{1}{q}}, \quad p>1, \quad \frac{1}{p}+\frac{1}{q}=1 ; \\
\left\|\frac{\partial^{n+m} f}{\partial t^{n} \partial s^{m}}\right\|_{1} \sup _{(t, s) \in[a, b][c, d]}\left|K_{n}(x, t) S_{m}(y, s)\right| .
\end{array}\right.
\end{aligned}
$$

Now, from (3.3) and using (2.1),

$$
\begin{aligned}
& \int_{a}^{b} \int_{c}^{d}\left|K_{n}(x, t) S_{m}(y, s)\right| d t d s \\
= & \int_{a}^{b}\left|K_{n}(x, t)\right| d t \int_{c}^{d}\left|S_{m}(y, s)\right| d s \\
= & {\left[\int_{a}^{x} \frac{(t-a)^{n}}{n !} d t+\int_{x}^{b} \frac{(b-t)^{n}}{n !} d t\right] \times\left[\int_{c}^{y} \frac{(s-c)^{m}}{m !} d s+\int_{y}^{d} \frac{(d-s)^{m}}{m !} d s\right] } \\
= & \frac{\left[(x-a)^{n+1}+(b-x)^{n+1}\right]\left[(y-c)^{m+1}+(d-y)^{m+1}\right]}{(n+1) !(m+1) !}
\end{aligned}
$$

giving the first inequality in (3.1).

Further, on using (2.1) and from (3.3)

$$
\begin{aligned}
& \left(\int_{a}^{b} \int_{c}^{d}\left|K_{n}(x, t) S_{m}(y, s)\right|^{q} d s d t\right)^{\frac{1}{q}} \\
= & \left(\int_{a}^{b}\left|K_{n}(x, t)\right|^{q} d t\right)^{\frac{1}{q}}\left(\int_{c}^{d}\left|S_{m}(y, s)\right|^{q} d s d t\right)^{\frac{1}{q}} \\
= & \frac{1}{n ! m !}\left[\int_{a}^{x}(t-a)^{n q} d t+\int_{x}^{b}(b-t)^{n q} d t\right]^{\frac{1}{q}} \times\left[\int_{c}^{y}(s-c)^{m q} d s+\int_{y}^{d}(d-s)^{m q} d s\right]^{\frac{1}{q}} \\
= & \frac{1}{n ! m !}\left[\frac{(x-a)^{n q+1}+(b-x)^{n q+1}}{n q+1}\right]^{\frac{1}{q}} \times\left[\frac{(y-c)^{m q+1}+(d-y)^{m q+1}}{m q+1}\right]^{\frac{1}{q}}
\end{aligned}
$$

producing the second inequality in (3.1).

Finally, from (2.1) and (3.3),

$$
\begin{aligned}
& \sup _{(t, s) \in[a, b] \times[c, d]}\left|K_{n}(x, t) S_{m}(y, s)\right| \\
= & \sup _{t \in[a, b]}\left|K_{n}(x, t)\right| \sup _{s \in[c, d]}\left|S_{m}(y, s)\right|
\end{aligned}
$$




$$
\begin{aligned}
= & \max \left\{\frac{(x-a)^{n}}{n !}, \frac{(b-x)^{n}}{n !}\right\} \times \max \left\{\frac{(y-c)^{m}}{m !}, \frac{(d-y)^{m}}{m !}\right\} \\
= & \frac{1}{n ! m !}\left[\frac{(x-a)^{n}+(b-x)^{n}}{2}+\left|\frac{(x-a)^{n}-(b-x)^{n}}{2}\right|\right] \\
& \times\left[\frac{(y-c)^{m}+(d-y)^{m}}{2}+\left|\frac{(y-c)^{m}+(d-y)^{m}}{2}\right|\right] .
\end{aligned}
$$

gives the inequality in (3.1) where we have used the fact that

$$
\max \{X, Y\}=\frac{X+Y}{2}+\left|\frac{Y-X}{2}\right| .
$$

Thus the theorem is now completely proved.

Keeping in mind that $x$ and $y$ are free parameters, we can produce "mid-point" and "boundary-point" type results by choosing appropriate values for $x$ and $y$. In addition, choosing values for $n$ and $m$ will recapture the earlier result of Hanna et al. [5] and Dragomir et al. [6].

From the results of Theorem 3 above, we have the following corollary.

Corollary 3. With the assumptions of Theorem 3, we have the inequality

$$
\begin{aligned}
& \mid \int_{a}^{b} \int_{c}^{d} f(t, s) d s d t-\sum_{k=0}^{n-1} \sum_{l=0}^{m-1} X_{k}\left(\frac{a+b}{2}\right) Y_{l}\left(\frac{c+d}{2}\right) \frac{\partial^{l+k}}{\partial t^{k} \partial s^{l}} f\left(\frac{a+b}{2}, \frac{c+d}{2}\right) \\
& -(-1)^{m} \sum_{k=0}^{n-1} X_{k}\left(\frac{a+b}{2}\right) \int_{c}^{d} \tilde{S}_{m}(s) \frac{\partial^{k+m}}{\partial t^{k} \partial s^{m}} f\left(\frac{a+b}{2}, s\right) d s \\
& -(-1)^{n} \sum_{l=0}^{m-1} Y_{l}\left(\frac{c+d}{2}\right) \int_{a}^{b} \tilde{K}_{n}(t) \frac{\partial^{n+l}}{\partial t^{n} \partial s^{l}} f\left(t, \frac{c+d}{2}\right) d t \mid \\
& \leq\left\{\begin{array}{l}
\frac{1}{2^{n+m}(n+1) !(m+1) !}(b-a)^{n+1}(d-c)^{m+1} \times\left\|\frac{\partial^{n+m} f}{\partial t^{n} \partial s^{m}}\right\|_{\infty} ; \\
\frac{1}{2^{n+m} n ! m !}\left[\frac{(b-a)^{n q+1}(d-c)^{m q+1}}{(n q+1)(m q+1)}\right]^{\frac{1}{q}} \times\left\|\frac{\partial^{n+m} f}{\partial t^{n} \partial s^{m}}\right\|_{p} ; \\
\frac{1}{2^{n+m} n ! m !}(b-a)^{n}(d-c)^{m} \times\left\|\frac{\partial^{n+m} f}{\partial t^{n} \partial s^{m}}\right\|_{1},
\end{array}\right.
\end{aligned}
$$

where $\|\cdot\|_{p}(p \in[1, \infty])$ are the Lebesgue norms on $[a, b] \times[c, d]$.

Proof. Taking $x=\frac{a+b}{2}$ and $y=\frac{c+d}{2}$ in (3.1) readily produces the result as stated.

These are the tightest possible for their respective Lebesgue norms, because of the symmetric and convex nature of the bounds in (3.1). 
Remark 1. For $n=m=1$ in (3.4) and $\frac{\partial^{2} f}{\partial t \partial s}$ belonging to the appropriate Lebesgue spaces on $[a, b] \times[c, d]$, we have

$$
\begin{aligned}
& \mid \int_{a}^{b} \int_{c}^{d} f(t, s) d s d t-(b-a)(d-c) f\left(\frac{a+b}{2}, \frac{c+d}{2}\right) . \\
& \quad+(b-a) \int_{c}^{d} \tilde{S}_{1}(s) \frac{\partial}{\partial s} f\left(\frac{a+b}{2}, s\right) d s+(d-c) \int_{a}^{b} \tilde{K}_{1}(t) \frac{\partial}{\partial t} f\left(t, \frac{c+d}{2}\right) d t \mid \\
& \leq\left\{\begin{array}{l}
\frac{1}{16}(b-a)^{2}(d-c)^{2} \times\left\|\frac{\partial^{2} f}{\partial t \partial s}\right\|_{\infty} ; \\
\frac{1}{4}\left[\frac{(b-a)^{q+1}(d-c)^{q+1}}{(q+1)^{2}}\right]^{\frac{1}{q}} \times\left\|\frac{\partial^{2} f}{\partial t \partial s}\right\|_{p} \\
\frac{1}{4}(b-a)(d-c) \times\left\|\frac{\partial^{2} f}{\partial t \partial s}\right\|_{1},
\end{array}\right.
\end{aligned}
$$

and thus some of the results of [5], [9] and [6] are recaptured.

Corollary 4. With the assumptions on $f$ as outlined in Theorem 3, we can obtain another result which is a generalization of the Trapezoid inequality

$$
\begin{aligned}
& \mid \int_{a}^{b} \int_{c}^{d} f(t, s) d s d t-\sum_{k=0}^{n-1} \sum_{l=0}^{m-1} \frac{(b-a)^{k+1}}{(k+1) !} \cdot \frac{(d-c)^{l+1}}{(l+1) !} \\
& \quad \times \frac{\partial^{k+l}}{\partial t^{k} \partial s^{l}}\left[\frac{f(a, c)+(-1)^{l} f(a, d)+(-1)^{k} f(b, c)+(-1)^{k+l} f(b, d)}{4}\right] \\
& -(-1)^{m} \sum_{k=0}^{n-1} \frac{(b-a)^{k+1}}{(k+1) !} \int_{c}^{d} \frac{(s-c)^{m}+(s-d)^{m}}{m !} \frac{\partial^{k+m}}{\partial t^{k} \partial s^{m}}\left[\frac{f(a, s)+(-1)^{k} f(b, s)}{4}\right] d s \\
& -(-1)^{n} \sum_{l=0}^{m-1}\left[\frac{(d-c)^{l+1}}{(l+1) !}\right] \int_{a}^{b} \frac{(t-a)^{n}+(t-b)^{n}}{n !} \frac{\partial^{l+n}}{\partial t^{n} \partial s^{l}}\left[\frac{f(t, c)+(-1)^{l} f(t, d)}{4}\right] d t \mid \\
& \leq\left\{\begin{array}{l}
\mathcal{K}_{n, m} \frac{(b-a)^{n+1}(d-c)^{m+1}}{(n+1) !(m+1) !}\left\|\frac{\partial^{n+m} f}{\partial t^{n} \partial s^{m}}\right\|_{\infty} ; \quad \text { if } \frac{\partial^{n+m} f}{\partial t^{n} \partial s^{m}} \in L_{\infty}([a, b] \times[c, d]) ; \\
\left.\left\|\frac{\partial^{n+m} f}{\partial t^{n} \partial s^{m}}\right\| \int_{p}^{b}\left|T_{n}(a, b ; t)\right|^{q} d t\right)^{\frac{1}{q}}\left(\int_{c}^{d}\left|T_{m}(c, d ; s)\right|^{q} d s\right)^{\frac{1}{q}} \\
\text { if } \frac{\partial^{n+m} f}{\partial t^{n} \partial s^{m}} \in L_{p}([a, b] \times[c, d]), p>1, \frac{1}{p}+\frac{1}{q}=1 ; \\
\left.\frac{(b-a)^{n}(d-c)^{m}}{4 n ! m !}\left\|\frac{\partial^{n+m} f}{\partial t^{n} \partial s^{m}}\right\|\right|_{1}, \quad \text { if } \frac{\partial^{n+m} f}{\partial t^{n} \partial s^{m}} \in L_{1}([a, b] \times[c, d]) .
\end{array}\right.
\end{aligned}
$$


where

$$
\mathcal{K}_{n, m}:= \begin{cases}1 & \text { if } n=2 r_{1} \text { and } m=2 r_{2}, \\ \frac{2^{n}-1}{2^{n}} & \text { if } n=2 r_{1}+1 \text { and } m=2 r_{2}, \\ \frac{2^{m}-1}{2^{m}} & \text { if } n=2 r_{1} \text { and } m=2 r_{2}+1, \\ \frac{\left(2^{n}-1\right)}{2^{n}} \cdot \frac{\left(2^{m}-1\right)}{2^{m}} & \text { if } n=2 r_{1}+1 \text { and } m=2 r_{2}+1\end{cases}
$$

where

$$
T_{n}(a, b ; t)=\left[\frac{(b-t)^{n}+(-1)^{n}(t-a)^{n}}{2 n !}\right], \quad T_{m}(c, d ; s)=\left[\frac{(d-s)^{m}+(-1)^{m}(s-c)^{m}}{2 m !}\right] .
$$

Proof. Using the identity (2.10), we find that

$$
\begin{aligned}
& \mid \int_{a}^{b} \int_{c}^{d} f(t, s) d s d t-\sum_{k=0}^{n-1} \sum_{l=0}^{m-1} \frac{(b-a)^{k+1}}{(k+1) !} \cdot \frac{(d-c)^{l+1}}{(l+1) !} \\
& \times \frac{\partial^{l+k}}{\partial x^{k} \partial y^{l}}\left[\frac{f(a, c)+(-1)^{l} f(a, d)+(-1)^{k} f(b, c)+(-1)^{k+l} f(b, d)}{4}\right] \\
& -(-1)^{m} \sum_{k=0}^{n-1} \frac{(b-a)^{k+1}}{(k+1) !} \int_{c}^{d} \frac{(s-c)^{m}+(s-d)^{m}}{m !} \times \frac{\partial^{k+m}}{\partial x^{k} \partial s^{m}}\left[\frac{f(a, s)+(-1)^{k} f(b, s)}{4}\right] d s \\
& -(-1)^{n} \sum_{l=0}^{m-1}\left[\frac{(d-c)^{l+1}}{(l+1) !}\right] \int_{a}^{b} \frac{(t-a)^{n}+(t-b)^{n}}{n !} \times \frac{\partial^{n+l}}{\partial y^{l} \partial t^{n}}\left[\frac{f(t, c)+(-1)^{l} f(t, d)}{4}\right] d t \mid \\
& =\left|\int_{a}^{b} \int_{c}^{d} T_{n}(a, b ; t) T_{m}(c, d ; s) \frac{\partial^{n+m} f}{\partial t^{n} \partial s^{m}} d s d t\right| \\
& \int\left\|\frac{\partial^{n+m} f}{\partial t^{n} \partial s^{m}}\right\|_{\infty} \int_{a}^{b} \int_{c}^{d}\left|T_{n}(a, b ; t) T_{m}(c, d ; s)\right| d t d s \text { if } \frac{\partial^{n+m} f}{\partial t^{n} \partial s^{m}} \in L_{\infty}([a, b] \times[c, d]) ; \\
& \leq\left\{\begin{array}{l}
\left\|\frac{\partial^{n+m} f}{\partial t^{n} \partial s^{m}}\right\|_{p}\left(\int_{a}^{b}\left|T_{n}(a, b ; t)\right|^{q} d t\right)^{\frac{1}{q}}\left(\int_{c}^{d}\left|T_{m}(c, d ; s)\right|^{q} d s\right)^{\frac{1}{q}} \\
\partial^{n+m} f
\end{array}\right. \\
& \leq\left\{\text { if } \frac{\partial^{n+m} f}{\partial t^{n} \partial s^{m}} \in L_{p}([a, b] \times[c, d]), p>1, \frac{1}{p}+\frac{1}{q}=1 ;\right. \\
& \left\|\frac{\partial^{n+m} f}{\partial t^{n} \partial s^{m}}\right\|_{1} \sup _{(t, s) \in[a, b] \times[c, d]}\left|T_{n}(a, b ; t) T_{m}(c, d ; s)\right| \text { if } \frac{\partial^{n+m} f}{\partial t^{n} \partial s^{m}} \in L_{1}([a, b] \times[c, d]) .
\end{aligned}
$$

Now consider $\int_{a}^{b}\left|T_{n}(a, b ; t)\right| d t$. As may be seen, explicit evaluation of the integral depends on whether $n$ is even or odd. 
(i) If $n$ is even, put $n=2 r_{1}$. Therefore,

$$
\begin{aligned}
\int_{a}^{b}\left|T_{n}(a, b ; t)\right| d t & =\frac{1}{\left(2 r_{1}\right) !} \int_{a}^{b} \frac{(b-t)^{2 r_{1}}+(t-a)^{2 r_{1}}}{2} d t \\
& =\frac{1}{\left(2 r_{1}\right) !} \cdot \frac{1}{2}\left[\frac{(b-a)^{2 r_{1}+1}}{2 r_{1}+1}+\frac{(b-a)^{2 r_{1}+1}}{2 r_{1}+1}\right] \\
& =\frac{(b-a)^{2 r_{1}+1}}{\left(2 r_{1}+1\right) !}=\frac{(b-a)^{n+1}}{(n+1) !} .
\end{aligned}
$$

Similarly,

$$
\int_{c}^{d}\left|T_{m}(c, d ; s)\right| d s=\frac{1}{\left(2 r_{2}\right) !} \int_{c}^{d} \frac{(d-s)^{2 r_{2}}+(s-c)^{2 r_{2}}}{2} d s=\frac{(d-c)^{m+1}}{(m+1) !}, \quad n \text { even. }
$$

(ii) Now, if $n$ is odd, that is, $n=2 r_{1}+1$, then

$$
T_{n}(a, b ; t)=\frac{(b-t)^{2 r_{1}+1}-(t-a)^{2 r_{1}+1}}{2\left(2 r_{1}+1\right) !} .
$$

Let $g(t)=(b-t)^{2 r_{1}+1}-(t-a)^{2 r_{1}+1}$.

We can observe that

$$
\begin{cases}g(t)<0 & \text { for all } t \in\left(\frac{a+b}{2}, b\right] \\ g(t)=0 & \text { at } t=\frac{a+b}{2} \\ g(t)>0 & \text { for all } t \in\left[a, \frac{a+b}{2}\right) .\end{cases}
$$

Thus

$$
\begin{aligned}
2\left(2 r_{1}+1\right) ! \int_{a}^{b}\left|T_{n}(a, b ; t)\right| d t= & {\left[\int_{a}^{\frac{a+b}{2}}\left[(b-t)^{2 r_{1}+1}-(t-a)^{2 r_{1}+1}\right] d t .\right.} \\
& \left.+\int_{\frac{a+b}{2}}^{b}\left[(t-a)^{2 r_{1}+1}-(b-t)^{2 r_{1}+1}\right] d t\right] \\
= & {\left[2 \cdot \frac{(b-a)^{2 r_{1}+2}}{2 r_{1}+2}-4 \frac{\left(\frac{b-a}{2}\right)^{2 r_{1}+2}}{2 r_{1}+2}\right] }
\end{aligned}
$$

and so

$$
\begin{aligned}
\int_{a}^{b}\left|T_{n}(a, b ; t)\right| d t & =\frac{(b-a)^{2 r_{1}+2}}{\left(2 r_{1}+2\right)\left(2 r_{1}+1\right) !}\left[1-\frac{1}{2^{2 r_{1}+1}}\right] \\
& =\frac{(b-a)^{2 r_{1}+2}}{\left(2 r_{1}+2\right) !}\left[\frac{2^{2 r_{1}+1}-1}{2^{2 r_{1}+1}}\right]=\frac{(b-a)^{n+1}}{(n+1) !}\left[\frac{2^{n}-1}{2^{n}}\right] .
\end{aligned}
$$


Similarly,

$$
\int_{c}^{d}\left|T_{m}(c, d ; s)\right| d s=\frac{(d-c)^{m+1}}{(m+1) !}\left[\frac{2^{m}-1}{2^{m}}\right], \quad m \text { even }
$$

and this gives the first inequality in (3.6).

Now, for the third inequality we have,

$$
\sup _{t \in[a, b]}\left|T_{n}(a, b ; t)\right|=\frac{1}{2 n !} \times \begin{cases}\sup _{t \in[a, b]}\left((b-t)^{n}+(t-a)^{n}\right)=\frac{(b-a)^{n}}{2 n !} & \text { for all } n \text { even } \\ \sup _{t \in[a, b]}\left|(b-t)^{n}-(t-a)^{n}\right|=\frac{(b-a)^{n}}{2 n !} & \text { for all } n \text { odd }\end{cases}
$$

and this gives last part of the inequality in (3.6). The corollary is thus completely proved.

Remark 2. For $n=m=1$, we have that

$$
\begin{aligned}
& \mid \int_{a}^{b} \int_{c}^{d} f(t, s) d s d t+\frac{(b-a)(d-c)}{4}[f(a, c)+f(a, d)+f(b, c)+f(b, d)] \\
& -\frac{b-a}{2}\left[\int_{c}^{d}(f(a, s)+f(b, s)) d s\right]-\frac{d-c}{2}\left[\int_{a}^{b}(f(t, c)+f(t, d)) d t\right] \mid \\
& \quad \leq\left\{\begin{array}{l}
\frac{(b-a)^{2}(d-c)^{2}}{16} \times\left\|\frac{\partial^{2} f}{\partial t \partial s}\right\|_{\infty} \\
\frac{1}{4}\left[\frac{((b-a)(d-c))^{q+1}}{(q+1)^{2}}\right]^{\frac{1}{q}} \times\left\|\frac{\partial^{2} f}{\partial t \partial s}\right\|_{p}, \quad p>1, \quad \frac{1}{p}+\frac{1}{q}=1 \\
\frac{(b-a)(d-c)}{4}\left\|\frac{\partial^{2} f}{\partial t \partial s}\right\|_{1} .
\end{array}\right.
\end{aligned}
$$

Again, the same result was obtained by G. Hanna et al. in [5] and S. Dragomir et al. in $[6]$.

In the following section we will utilise the inequalities obtained in this section and demonstrate their capabilities to numerical integration.

\section{Applications to Numerical Integration}

Consider $I$ to be a two-dimensional, $n$-times differentiable mapping, where all partial derivatives in both directions exist and are integrable. We apply the inequalities obtained in Section 3 for numerical implementation using a uniform mesh. The following application in Numerical Integration is natural to be considered.

Theorem 4. Let $f:[a, b] \times[c, d] \rightarrow \mathbb{R}$ be as in Theorem 3. In addition, let $I_{v}$ and $J_{\mu}$ be arbitrary divisions of $[a, b]$ and $[c, d]$ respectively, that is,

$$
I_{v}: a=\xi_{0}<\xi_{1}<\cdots<\xi_{\nu}=b,
$$


where $x_{i} \in\left(\xi_{i}, \xi_{i+1}\right)$ for $i=0,1, \ldots, \nu-1$, and

$$
J_{\mu}: c=\tau_{0}<\tau_{1}<\cdots<\tau_{\mu}=d,
$$

with $y_{j} \in\left(\tau_{j}, \tau_{j+1}\right)$ for $j=0,1, \ldots, \mu-1$, then we have the cubature formula

$$
\begin{aligned}
& \int_{a}^{b} \int_{c}^{d} f(t, s) d s d t \\
= & \sum_{k=0}^{n-1} \sum_{l=0}^{m-1} \sum_{i=0}^{\nu-1} \sum_{j=0}^{\mu-1} X_{k}^{(i)}\left(x_{i}\right) Y_{l}^{(j)}\left(y_{j}\right) \frac{\partial^{i+j} f\left(x_{i}, y_{j}\right)}{\partial x^{i} \partial y^{j}} \\
& +(-1)^{m} \sum_{k=0}^{n-1} \sum_{i=0}^{\nu-1} \sum_{j=0}^{\mu-1} X_{k}^{(i)}\left(x_{i}\right) \int_{\tau_{j}}^{\tau_{j+1}} S_{m}^{(j)}\left(y_{j}, s\right) \frac{\partial^{k+m} f\left(x_{i}, s\right)}{\partial x^{k} \partial s^{m}} d s \\
& +(-1)^{n} \sum_{l=0}^{m-1} \sum_{i=0}^{\nu-1} \sum_{j=0}^{\mu-1} Y_{l}^{(j)}\left(y_{j}\right) \int_{\xi_{i}}^{\xi_{i+1}} K_{n}^{(i)}\left(x_{i}, t\right) \frac{\partial^{n+l} f\left(t, y_{j}\right)}{\partial t^{n} \partial y^{l}} d t \\
& +R\left(f, I_{v}, J_{\mu}, x, y\right),
\end{aligned}
$$

where the remainder term satisfies the condition

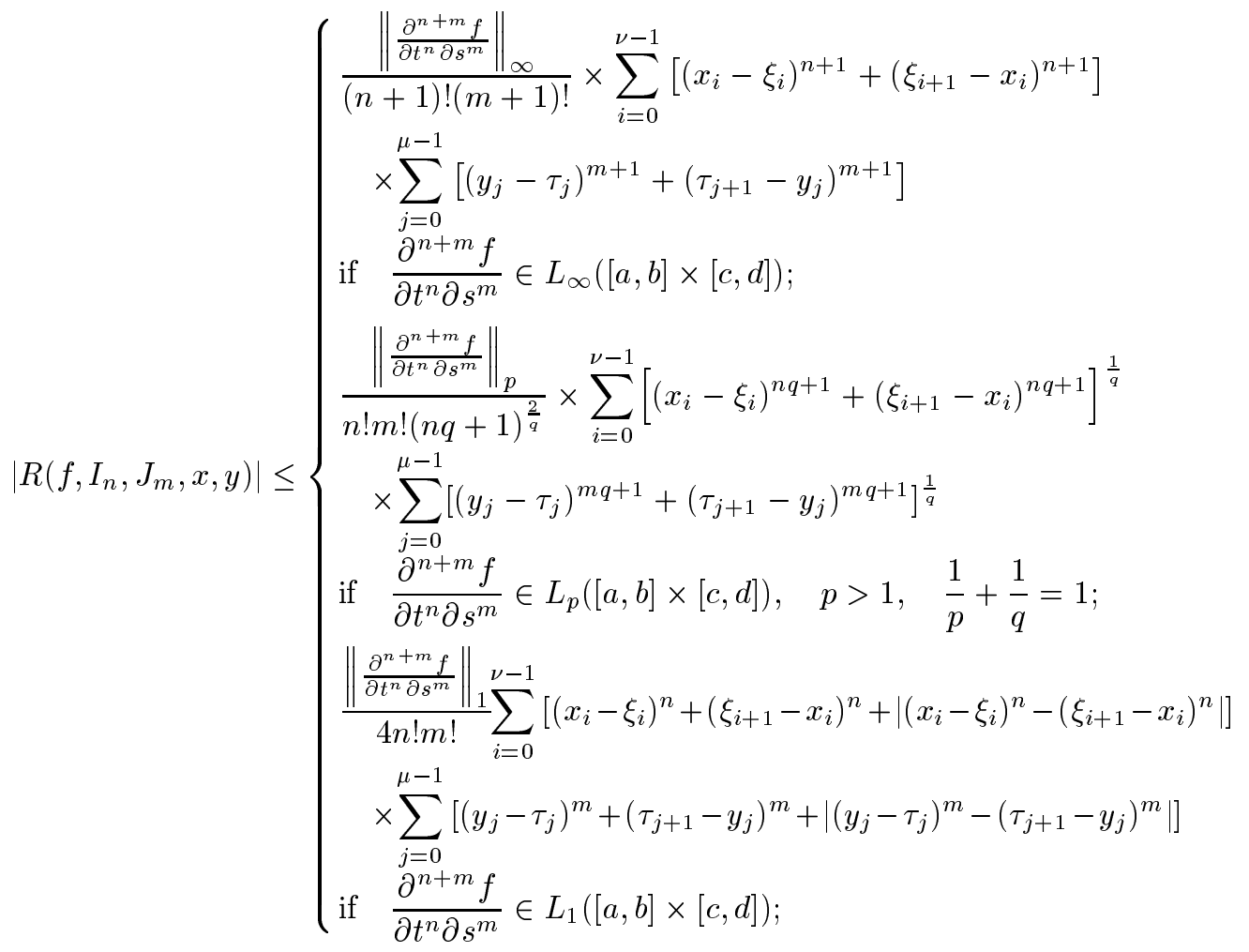


where

$X_{k}^{(i)}(k=0,1, \ldots, n-1 ; i=0,1, \ldots, \nu-1), \quad Y_{l}^{(j)}(l=0,1, \ldots, m-1 ; j=0,1, \ldots, \mu-1)$

and

$$
\begin{aligned}
K_{n}^{(i)}(i=0,1, \ldots, \nu & -1), S_{m}^{(j)}(j=0,1, \ldots, \mu-1) \text { are defined by } \\
X_{k}^{(i)}\left(x_{i}\right): & =\frac{\left(\xi_{i+1}-x_{i}\right)^{k+1}+(-1)^{k}\left(x_{i}-\xi_{i}\right)^{k+1}}{(k+1) !} \\
Y_{l}^{(j)}\left(y_{j}\right): & =\frac{\left(\tau_{j+1}-y_{j}\right)^{l+1}+(-1)^{l}\left(y_{j}-\tau_{j}\right)^{l+1}}{(l+1) !}, \\
K_{n}^{(i)}\left(x_{i}, t\right) & := \begin{cases}\frac{\left(t-\xi_{i}\right)^{n}}{n !}, & t \in\left[\xi_{i}, x_{i}\right] \\
\frac{\left(t-\xi_{i+1}\right)^{n}}{n !}, & t \in\left(x_{i}, \xi_{i+1}\right]\end{cases} \\
S_{m}^{(j)}\left(y_{j}, s\right) & := \begin{cases}\frac{\left(s-\tau_{j}\right)^{m}}{m !}, & s \in\left[\tau_{i}, y_{i}\right] \\
\frac{\left(s-\tau_{j+1}\right)^{m}}{m !}, & s \in\left(y_{i}, \tau_{j+1}\right] .\end{cases}
\end{aligned}
$$

The proof is obvious by Theorem 3 applied on the interval $\left[\xi_{i}, \xi_{i+1}\right] \times\left[\tau_{j}, \tau_{j+1}\right]$, $(i=0,1, \ldots, \nu-1 ; j=0,1, \ldots, \mu-1)$, and we omit the details.

Remark 3. Similar result can be obtained if we use the other results obtained in section 3, but we omit the details.

\section{References}

[1] P. Cerone, S. S. Dragomir and J. Roumeliotis, Some Ostrowski type inequalities for n-time differentiable mappings and applications, Demonstratio Math. 32 (1999), 697-712.

[2] D. S. Mitrinović, J. E. Pečarić and A. M. Fink, Inequalities for Functions and Their Integrals and Derivatives, Kluwer Academic, Dordrecht, 1994.

[3] N. S. Barnett and S. S. Dragomir, An Ostrowski type inequality for double integrals and application for cubature formulae, Soochow J. of Math., 27 (2001), 1-10.

[4] S. S. Dragomir, N. S. Barnett and P. Cerone, An Ostrowski type inequality for double integrals in terms of $L_{p}$ - Norms and applications in Numerical integrations, Anal. Num. Theor. Approx (Romania), (accepted).

[5] G. Hanna, P. Cerone and J. Roumeliotis, An Ostrowski type inequality in two dimensions using the three point rule, CTAC 'Computational Techniques and Applications Conference.

[6] S. S. Dragomir, P. Cerone, N. S. Barnett and J. Roumeliotis, An inequality of the Ostrowski type for double integrals and applications for cubature formulae, Tamsui Oxford J. Math. 16 (2000), 1-16. 
[7] A. M. Fink, Bounds on the derivation of a function from its averages, Czechoslovak Math. J. 42 (1992), 289-310.

[8] G. A. Anastassiou, Ostrowski type inequalities, Proc. Amer. Math. Soc. 123(1995), 37753781.

[9] B. G. Pachpatte, On a new Ostrowski type inequality in two independent variables, Tamkang J. Math. 32 (2001), 45-49.

School of Communications and Informatics, Victoria University of Technology, PO Box 14428, Melbourne City Mc, Victoria 8001, Australia.

E-mail: georgey@sci.vu.edu.au

E-mail: sever.dragomir@vu.edu.au

URL: http://rgmia.vu.edu.au/SSDragomirWeb.html

E-mail: pc@sci.vu.edu.au 